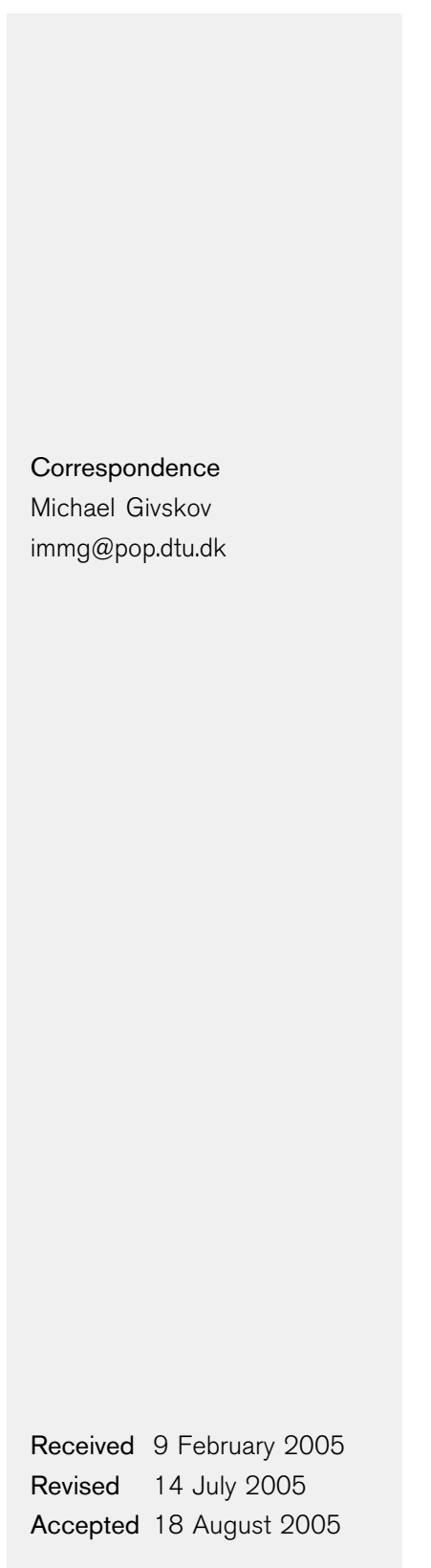

\title{
Garlic blocks quorum sensing and promotes rapid clearing of pulmonary Pseudomonas aeruginosa infections
}

\author{
Thomas Bjarnsholt, ${ }^{1}$ Peter Østrup Jensen, ${ }^{2}$ Thomas B. Rasmussen, ${ }^{1}$ \\ Lars Christophersen, ${ }^{2}$ Henrik Calum, ${ }^{2}$ Morten Hentzer, ${ }^{3}$ \\ Hans-Petter Hougen, ${ }^{4}$ Jørgen Rygaard, ${ }^{5}$ Claus Moser, ${ }^{2}$ Leo Eberl, ${ }^{6}$ \\ Niels Høiby ${ }^{2}$ and Michael Givskov ${ }^{1}$ \\ ${ }^{1}$ Centre for Biomedical Microbiology, BioCentrum, Technical University of Denmark, DK-2800 \\ Lyngby, Denmark \\ 2Department of Clinical Microbiology, Rigshospitalet, DK-2100 Copenhagen $\varnothing$, Denmark \\ ${ }^{3}$ Carlsberg Research Center, Biosector, Gamle Carlsberg Vej 10, DK-2500 Valby, Denmark \\ ${ }^{4}$ Institute of Forensic Medicine, University of Copenhagen, DK-2100 Copenhagen $\varnothing$, Denmark \\ ${ }^{5}$ Bartholin Instituttet, Kommune Hospitalet, Copenhagen, Denmark \\ ${ }^{6}$ Department of Microbiology, University of Zürich, $\mathrm{CH}-8008$ Zürich, Switzerland
}

The opportunistic human pathogen Pseudomonas aeruginosa is the predominant micro-organism of chronic lung infections in cystic fibrosis patients. $P$. aeruginosa colonizes the lungs by forming biofilm microcolonies throughout the lung. Quorum sensing (QS) renders the biofilm bacteria highly tolerant to otherwise lethal doses of antibiotics, and protects against the bactericidal activity of polymorphonuclear leukocytes (PMNs). It has been previously demonstrated that $\mathrm{QS}$ is inhibited by garlic extract. In this study, the synergistic effects of garlic and tobramycin, and PMNs activities have been evaluated. P. aeruginosa was grown in vitro in continuous-culture once-through flow chambers with and without garlic extract. The garlic-treated biofilms were susceptible to both tobramycin and PMN grazing. Furthermore, the PMNs showed an increase in respiratory burst activation, when incubated with the garlic-treated biofilm. Garlic extract was administered as treatment for a mouse pulmonary infection model. Mice were treated with garlic extract or placebo for 7 days, with the initial 2 days being prophylactic before $P$. aeruginosa was instilled in the left lung of the mice. Bacteriology, mortality, histopathology and cytokine production were used as indicators. The garlic treatment initially provoked a higher degree of inflammation, and significantly improved clearing of the infecting bacteria. The results indicate that a aS-inhibitory extract of garlic renders $P$. aeruginosa sensitive to tobramycin, respiratory burst and phagocytosis by PMNs, as well as leading to an improved outcome of pulmonary infections.

\section{INTRODUCTION}

Several opportunistic pathogens are capable of organized expression of their arsenal of virulence factors in eukaryotic hosts. In order to do this, the bacteria have developed a chemically based, command language referred to as quorum sensing (QS) (Fuqua et al., 1994). QS enables bacteria to keep track of their numbers, and is considered to afford them a mechanism for minimizing host responses by delaying the production of virulence factors until sufficient

Abbreviations: CF, cystic fibrosis; G-CSF, granulocyte colony-stimulating factor; GFP, green fluorescent protein; MN, monocyte; MPO, myeloperoxidase; PMN, polymorphonuclear leukocytes; OS, quorum sensing; OSI, quorum sensing inhibitor. bacteria have been amassed to overwhelm host defence mechanisms. Pseudomonas aeruginosa is a prevalent opportunistic human pathogen and one of the most common Gram-negative bacteria found in nosocomial and lifethreatening infections of immuno-compromised patients (van Delden \& Iglewski, 1998). Patients with cystic fibrosis (CF) are especially predisposed to infection with $P$. aeruginosa, and the bacterium is, despite intensive antibiotic therapy, responsible for high rates of morbidity and mortality (Lyczak et al., 2002; Koch \& Høiby, 2000). Alternative strategies to conventional antibiotic treatments are therefore required. Our recently published tool for high-throughput screening, the QS inhibitor (QSI)-selector has allowed us to identify natural sources of potential, non-toxic QSI 
compounds (Rasmussen et al., 2005). The presence of such compounds in certain natural foods is interesting since it suggests that the diet may, to some extent, offer a natural prophylaxis against chronic $P$. aeruginosa infections. Garlic is renowned for its anti-fungal, anti-cancer and antimicrobial activities. The anti-microbial activities have been related to the presence of growth-inhibitory compounds, such as allicin and related derivatives (Ankri \& Mirelman, 1999), and QS-blocking properties of garlic have been reported by us (Rasmussen et al., 2005, Persson et al., 2005).

We have previously demonstrated that a crude extract of garlic specifically inhibits QS-regulated gene expression in $P$. aeruginosa, as judged from DNA microarray-based transcriptomic analysis (Rasmussen et al., 2005). The expression of 167 genes, 92 of which were regulated by QS, was repressed by the extract (Rasmussen et al., 2005). The mechanism by which garlic compounds block QS is presently unknown. However, the effect is at a posttranscriptional level, since the amounts of mRNA of neither lasI, lasR, rhlI nor rhlR (the key components of the Las and Rhl QS communication systems in $P$. aeruginosa) were notably affected by the garlic treatment (Rasmussen et al. 2005; unpublished results). This suggests that the QSI molecules interact directly with the QS receptors by either a competitive or a non-competitive $\mathrm{N}$-acylhomoserine lactone mechanism. The garlic extract used in this study contained very low amounts of toxic compounds (such as allicin) (Rasmussen et al., 2005). Treatment of in vitro biofilms with this extract was found to dramatically reduce the tolerance of the bacteria to the antibiotic tobramycin (Rasmussen et al., 2005). In addition, the extract was capable of attenuating bacterial virulence in a Caenorhabditis elegans nematode infection model (Rasmussen et al., 2005). We have previously demonstrated that the QS system is in fact a useful drug target, and delivered a proof of concept in which we demonstrated that the blockade of QS with the drug C-30 represents an effective approach for interfering with biofilm tolerance to antibiotic treatments and for attenuating the virulence of $P$. aeruginosa in a mouse infection model ( $\mathrm{Wu}$ et al., 2004; Hentzer et al., 2003). Recently we have shown that the activation of polymorphonuclear leukocytes (PMNs) is blocked by $P$. aeruginosa QS signals in vitro (Bjarnsholt et al., 2005). In addition, QS mutants cause a faster activation of the host defence system in vivo (Bjarnsholt et al., 2005; Wu et al., 2001). These factors might account for the rapid clearing of bacteria deficient in QS (either by inactivating mutations or by treatment with QS-blocking drugs) as observed in mouse models of $P$. aeruginosa pulmonary infections (Wu et al., 2001, 2004; Hentzer et al., 2003; Bjarnsholt et al., 2005).

\section{METHODS}

Bacterial strains. The $P$. aeruginosa PAO1 strain used for the in vitro experiments with biofilms was obtained from the Pseudomonas Genetic Stock Center (www.ecu.edu/pseudomonas; strain PAO0001). This PAO1 isolate has served as the DNA source for the Pseudomonas Genome Project (www.pseudomonas.com) and, subsequently, as a template for the design of the $P$. aeruginosa GeneChip (Affymetrix). Construction of an isogenic $\Delta$ lasR $\Delta r h l R$ mutant was described previously (Bjarnsholt et al., 2005). For green fluorescent protein (GFP) tagging of the bacteria, we used the constitutively expressed, stable GFP on the plasmid pMRP9. The P. aeruginosa used for in vivo experiments was obtained from Professor B. Iglewski, University of Rochester Medical Center, New York, USA.

Preparation of garlic extract. The garlic extract used in all the experiments was prepared, evaluated and found to have no growth inhibitory effect on $P$. aeruginosa, as described previously (Rasmussen et al., 2005).

Biofilms. Biofilms were cultivated in continuous-culture oncethrough flow chambers, perfused with sterile ABtrace minimal medium containing $0.3 \mathrm{mM}$ glucose as described previously (Christensen et al., 1999). The pump was set to $3 \mathrm{ml} \mathrm{h}^{-1}$. Garlic extract $(2 \cdot 0 \%, \mathrm{v} / \mathrm{v})$ was added to medium as appropriate. The concentration of garlic extract used was the concentration that inhibits QS the most without inhibiting growth. The preparation and concentration calibration of the garlic extract was performed as described previously (Rasmussen et al., 2005). Biofilm development was examined by scanning confocal laser microscopy (SCLM) using a Zeiss LSM 510 system equipped with an argon laser and a heliumneon laser for excitation of fluorophores. Simulated projections and vertical cross-sections of the biofilms were generated with the Imaris software package (Bitplane). Images were further processed with Photoshop software (Adobe).

Preparation of PMNs. Human blood samples were obtained from normal healthy volunteers, by venous puncture, and collected in $\mathrm{BD}$ vacutainers coated with heparin and lithium (Becton-Dickinson, 388330). The blood was mixed with dextran (T-500) $1: 5$ and the erythrocytes were sedimented for $40 \mathrm{~min}$. The supernatant was applied to Lymphoprep (Axis-Shield) and centrifuged at 2200 r.p.m. for $15 \mathrm{~min}$ at $5{ }^{\circ} \mathrm{C}$. The supernatant was discarded and the neutrophils were treated with $2 \mathrm{ml} 0 \cdot 2 \% \mathrm{NaCl}$ in order to lyse remaining erythrocytes. Lysis was terminated by adding $2 \mathrm{ml} 1.6 \% \mathrm{NaCl}$ and $6 \mathrm{ml}$ Eagle-MEM (Bie \& Berntsen). The cells were centrifuged at $350 \mathrm{~g}$ for $10 \mathrm{~min}$ at $5^{\circ} \mathrm{C}$, the supernatant was discarded and the PMNs were resuspended in Eagle-MEM.

PMN treatment of biofilms. In order to inoculate PMNs into the biofilm chambers, the flow was stopped and the flow cells clamped off. PMNs in the order of $1.5 \times 10^{6}$ were inoculated in each flow channel. The flow cells were incubated top down in a $37^{\circ} \mathrm{C}$ water bath, with shaking, until microscopic inspection.

Monitoring the oxidative burst of PMNs. The PMNs were incubated for approximately $30 \mathrm{~min}$ in Eagle-MEM $\left(10 \times 10^{6} \mathrm{cells} \mathrm{ml}^{-1}\right)$ with $10 \%$ normal human $\mathrm{AB}$ serum, $5 \mu \mathrm{M}$ SYTO 62 (Molecular Probes), to stain the nuclei (dsDNA), and $0.1 \mathrm{mg} 123$-dihydrorhodamine $\mathrm{ml}^{-1}$ (Sigma), to stain the $\mathrm{H}_{2} \mathrm{O}_{2}$ in the phagosomes (Bassøe et al., 2003). A $100 \mu$ l quantity of the PMN mixture was added to each biofilm.

Animals. Female BALB/c mice were purchased from $M$ \& $B$ Laboratory Animals at 10 to 11 weeks of age. The mice were of equal size and were maintained on standard mouse chow and water ad libitum for one week before challenge. All animal experiments were authorised by the National Animal Ethics Committee.

Immobilization of $\boldsymbol{P}$. aeruginosa in seaweed alginate beads. Immobilization of $P$. aeruginosa in seaweed alginate beads was performed as described by Pedersen et al. (1990). The suspension was adjusted to $2.5 \times 10^{8}$ c.f.u. $\mathrm{ml}^{-1}$, confirmed by colony counts, and $0.04 \mathrm{ml}$ of the suspension was instilled in the left lung of each mouse.

Garlic extract administration. Each mouse was treated with $1.5 \%$ garlic extract $(\mathrm{kg} \text { body weight })^{-1}$. The garlic extract $(0 \cdot 3 \mathrm{ml})$ 
was mixed with $0.7 \mathrm{ml} 0.9 \% \mathrm{NaCl}$ and injected subcutaneously. The control groups had $1 \mathrm{ml} 0.9 \% \mathrm{NaCl}$ injected subcutaneously. The mice were treated two days prior to the bacterial challenge. The treatment was continued until the mice were sacrificed.

Challenge procedure. The mice were anaesthetized by subcutaneous injection of $0.2 \mathrm{ml}$ Hyp/Mid $\left[2.5 \mathrm{mg}\right.$ Hypnorm $\mathrm{ml}^{-1}$ (Janssen) and $1.25 \mathrm{mg}$ midazolam $\mathrm{ml}^{-1}$ (Roche) in sterile water]. Sedated mice were fixed and their tracheas were exposed and penetrated with an $18 \mathrm{G}$ needle. The inoculum was installed in the left lung approximately $11 \mathrm{~mm}$ from the tracheal penetration site with a curved bead-tipped needle (Moser et al., 1997). The incision was sutured with silk and healed without any complications. Pentobarbital (DAK), $2 \cdot 0 \mathrm{ml}$ (kg body weight $)^{-1}$, was used to sacrifice the animals. The mice were sacrificed at different time points after infection as mentioned in Results and Discussion.

Bacteriology. Lungs from mice were prepared for bacteriology examination as described by Moser et al. (1997). Isolated lungs were homogenized on ice. A serial dilution of the lung homogenate was performed and plated on blue agar plates (States Serum Institute), containing selective culture medium for Gram-negative bacilli, for colony counting.

ELISA measurements of cytokines and proteins. The concentration of the cytokines and proteins was measured in supernatant from lung homogenate by ELISA kits (G-CSF and MIP-2; R\&D systems) (GM-CSF, TNF- $\alpha$, IL-6; Becton Dickinson) (Myeloperoxidase, MPO; HyCult Biotechnology). The measurements were performed according to the manufacturer's recommendations.

Histopathology. For examination of histopathology, the isolated lungs were immediately fixed in PBS with $4 \%$ paraformaldehyde and kept at $5{ }^{\circ} \mathrm{C}$ before further preparation.

By microscopy, slides with two tissue sections mounted on each were used for evaluation. Based on an overview at low magnification $(\times 62.5)$, and detailed studies of five different areas of each tissue section under high magnification $(\times 500)$, the degree of inflammation was scored as 0 (no inflammation), 1 (mild inflammation), 2 (moderate to severe inflammation) and 3 (severe inflammation with necrosis or with severe inflammation throughout the lung). The histopathological evaluation was done blindly.

\section{RESULTS AND DISCUSSION}

\section{In vitro effect of garlic extract on PMNs}

Our previous results regarding $P$. aeruginosa tolerance to PMNs encouraged us to investigate whether the attenuation of QS regulation by a QSI also had an effect of the activity of PMNs. To investigate whether PMNs became activated upon exposure to garlic-extract-treated bacteria, two sets of $P$. aeruginosa wild-type biofilms were established and grown with or without the presence of $2 \%$ garlic extract in the influent medium. The concentration of garlic extract used in all the experiments in this study has no effect on the growth of the bacteria. The biofilms were allowed to develop for 3 days. On day 3, freshly isolated PMNs were inoculated as described previously (Bjarnsholt et al., 2005) into the flow chambers. As seen from Fig. 1, garlic-treated biofilm promoted development of an oxidative burst, whereas the PMNs were not activated when inoculated on the untreated biofilm. As a control, a $\Delta$ lasR rhlR QS-mutant biofilm grown in the presence or absence of garlic extract was exposed to

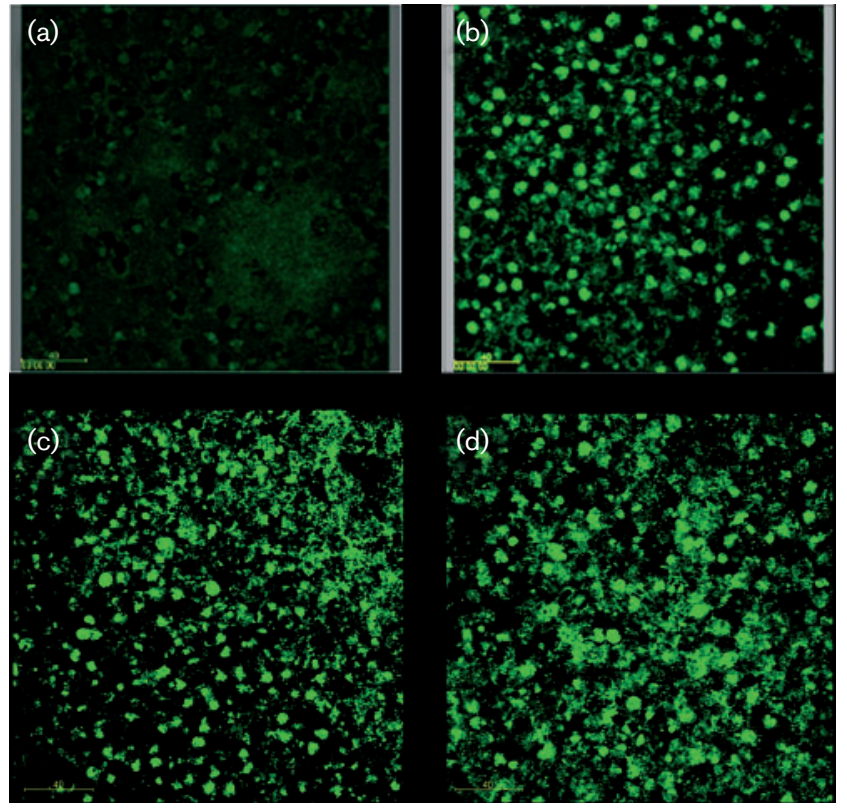

Fig. 1. Garlic-dependent activation of PMNs. SCLM micrographs of 3 day old biofilms of wild-type $P$. aeruginosa in the absence (a) and presence (b) of $2 \%$ garlic extract, and $\mathrm{OS}$ mutant $\Delta / a s R$ rh/R in the absence (c) and presence (d) of $2 \%$ garlic extract, exposed to $3 \times 10^{6}$ freshly prepared human PMNs for $2.5 \mathrm{~h}$ at $37^{\circ} \mathrm{C}$. Activated PMNs emit green fluorescence. No activation was detected when the PMNs were incubated on the wild-type biofilm in the absence of garlic extract, whereas the PMNs became activated on the garlic-extract-treated wildtype biofilm and the QS-deficient biofilm. In addition, the PMNs did not activate earlier or more on the garlic-extract-treated QSmutant biofilm compared to the untreated QS-mutant biofilm.

PMNs. No difference in intensity of the green fluorescence was detected between these two controls. We also tested the activation of PMNs in the presence of garlic extract without bacteria. No activation took place (results not shown). There are two possible explanations, or a combination thereof, to this observation: (1) garlic does not affect the PMNs, but solely blocks the bacterial QS system thereby promoting the activation of the PMNs, or (2) garlic directly affects the PMNs thereby promoting their activation in the presence of bacteria. Work is in progress to elucidate the effect of QS blockers on the PMN transcriptome.

\section{Grazing}

We repeated the above experiment with GFP-tagged $P$. aeruginosa to follow the fate of the biofilm upon PMN exposure. As seen from Fig. 2, the garlic-treated biofilm was grazed extensively by the PMNs in contrast to the untreated biofilm. This is in accordance with our previous findings where PMNs became activated, grazed extensively and phagocytosed the biofilm cells of $P$. aeruginosa QS-deficient mutants in contrast to their wild-type counterparts (Bjarnsholt et al., 2005). The in vitro experiments described 

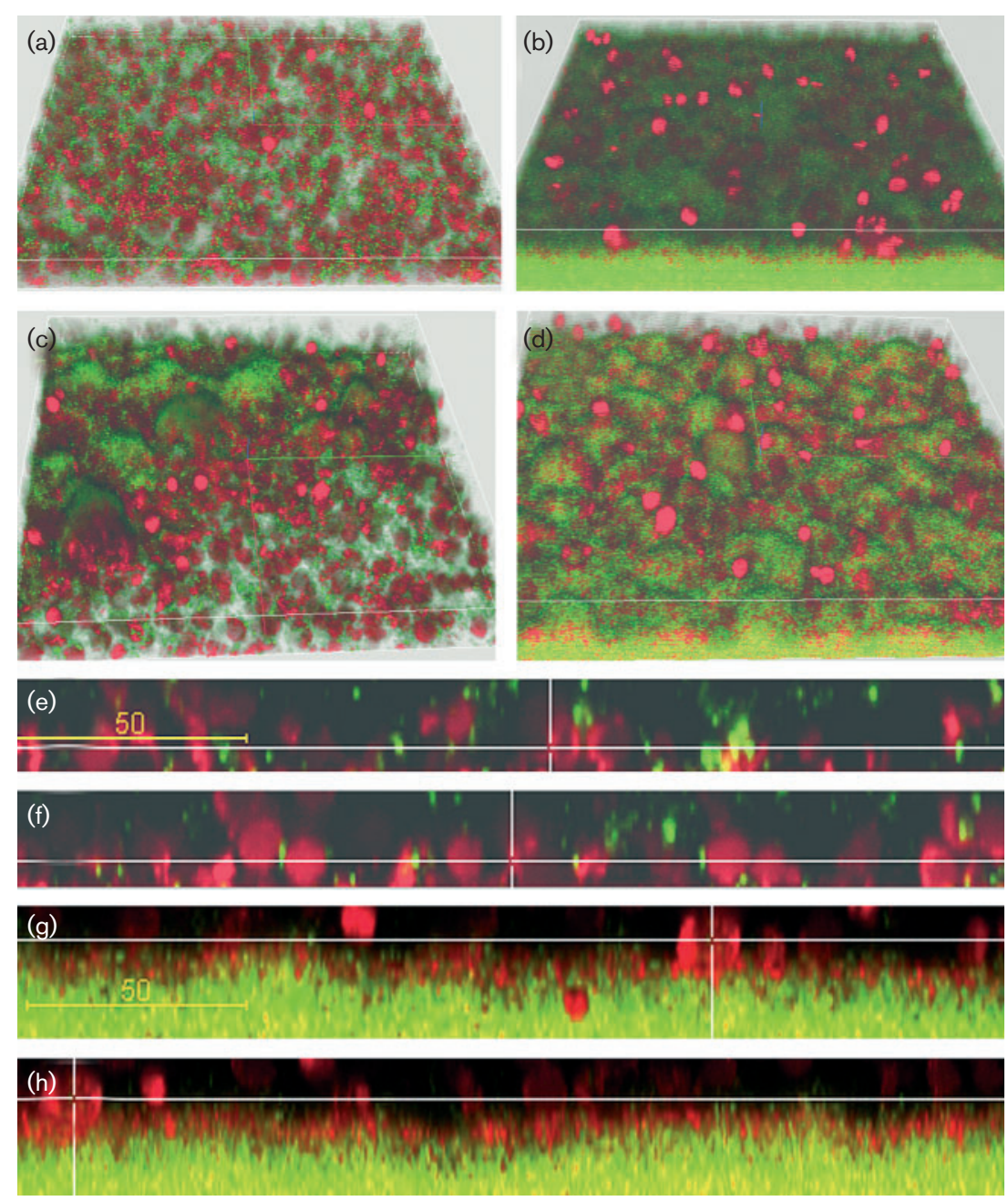

Fig. 2. Garlic-dependent sensitivity of $P$. aeruginosa biofilms towards PMNs. Two sets of biofilms of PAO1 cells (green) were grown in the presence or absence of $2 \%$ garlic extract. On day 3 the biofilms were exposed to PMNs (red) for 2.5 h. (a, c) 3D projections, and (e, f) cross-sections, showing the garlic-treated biofilm fully penetrated by PMNs. (b, d) 3D projections, and $(g, h)$ cross-sections, showing the untreated biofilm with PMNs on top. above (both for activation and grazing) have been repeated a minimum of three times producing similar results.

\section{In vivo effects of garlic extract}

In order to study the effect of the garlic extract on a pulmonary infection model (Moser et al., 1997), two groups of $\mathrm{BALB} / \mathrm{c}$ mice were treated with garlic extract or placebo (saline). The BALB/c mice are susceptible mice with a Th-2 dominated immune response (Moser et al., 1999), resembling the immune response of chronic $P$. aeruginosa-infected CF patients with poor prognosis (Moser et al., 2000). The treatments were given as single subcutaneous injections every $24 \mathrm{~h}$ for 7 days. The dosage of garlic extract given was $1.5 \%$ of the mass of the mouse $(20 \mathrm{~g})$. After two days of prophylactic treatment, the mice were intratracheally challenged with alginate beads containing $P$. aeruginosa, at $2 \times 10^{7}$ c.f.u. per lung. This challenge dose was too high for the investigation of clearance of the bacteria, since $23 / 32$ of the mice died in the placebo group in contrast to a mortality rate of $10 / 31$ in the group treated with garlic extract $\left(P<0 \cdot 003, \chi^{2}\right.$ test $)$. This indicates that the prophylactic garlic treatment significantly reduced mortality.
Clearance of the infecting bacteria was assessed in a similar experimental setup, but with a reduced bacterial challenge $\left(1 \times 10^{7}\right.$ c.f.u. per lung). Garlic and placebo treatments were given as described for the previous experiment. Mice were sacrificed on days 1, 3 and 5 post-challenge, and the bacterial content in the lungs of the animals was determined. On average, the garlic-treated group displayed three orders of magnitude fewer c.f.u. per lung. On day 5, the lungs of 9/10 mice in the garlic-treated group were sterile, whereas the placebo group still contained a mean of $1 \times 10^{5}$ c.f.u. per lung (Fig. 3).

\section{Garlic extract treatment provokes a higher degree of inflammation}

On days 1, 3, 5 and 7, mice were sacrificed for histopathological evaluation. The lungs were recovered and fixed in formalin. Sections (thickness $4 \mu \mathrm{m}$ ) were stained with haematoxylin/eosin for evaluation of pathological changes by light microscopy. On days 1,3 and 5 post-challenge, all mice showed histopathological changes in the lungs (Tables 1 and 2). Compared to the untreated group, a significantly higher degree of inflammation was detected on 


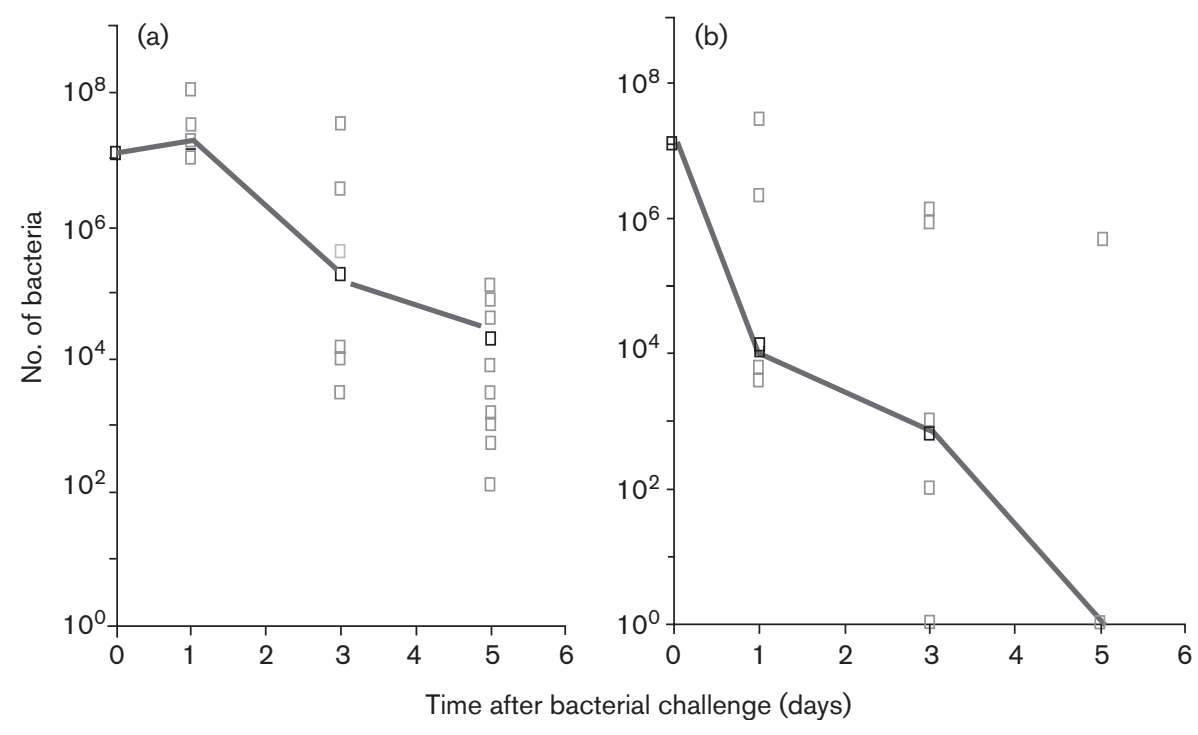

Fig. 3. In vivo clearance of P. aeruginosa promoted by garlic extract. Two groups of BALB/c mice were treated with placebo (saline) (a) or garlic extract (b). After 3 days of prophylactic treatment, the mice were intratracheally challenged with alginate beads containing $1 \times 10^{7}$ c.f.u. $P$. aeruginosa per lung. Each group contained, at the time of sacrifice, a minimum of 7 mice. Mice were sacrificed on days 1, 3 and 5 post-infection, and the content of bacteria in the lungs was determined. The statistical significance of the difference in clearance was measured by a Kruskal-Wallis test (analysis of nonparametric data) and $P$ values for the difference at days 1,3 and 5 were $0.0087,0.0636$ and 0.0015 , respectively. $\square$, Individual c.f.u. values, lines intercept medians.

day 5 in the group of mice treated with garlic extract $(P<0 \cdot 04)$ (Table 2). We also detected that the 13/14 lungs of mice treated with garlic contained PMNs and monocytes (MNs), in contrast only 4/13 lungs of the placebo-treated mice contained PMNs and MNs $(P<0 \cdot 0008)$. For the placebo group, $6 / 13$ of the mice lungs were dominated by MNs only (Table 1). This modulated acute immune response suggests that the garlic treatment counteracts the otherwise quorum-signal mediated attenuation of PMN activation as described recently (Bjarnsholt et al., 2005). Therefore, the innate immune defence responds to the presence of the infective bacteria more efficiently due to the QSblocking effect of the garlic extract. Accordingly, bacteria are efficiently cleared by the host defence system. This is in contrast to the placebo-treated group in which the bacterial QS signals block or delay PMN activation. This results in a significantly reduced bacterial clearance.

\section{Cytokines and proteins measured in the lungs}

To gain an insight into the difference between the immunological reaction of the treated and the untreated group of mice, we measured the following cytokines in the lung homogenate. Both granulocyte colony-stimulating factor (G-CSF) and macrophage inflammatory protein-2 (MIP-2) were down-regulated (Figs 4 and 5) in the garlic-extracttreated group. No differences in the concentration of granulocyte macrophage colony-stimulating factor (GM-CSF), tumour necrosis factor alpha (TNF- $\alpha$ ), interleukin 6 (IL-6) and MPO were detected.

MIP-2 is the mouse analogue of human IL-8, which functions as a chemoattractant for PMNs. In chronic $P$. aeruginosa-infected CF patients, IL-8 production in the lungs keeps the immune response acute and morbid because of constantly recruited PMNs (Chmiel \& Davis, 2003). It has been reported that the production of IL- 8 by respiratory epithelial cells is stimulated by the 3-oxo-dodecanoylhomoserine lactone quorum signal (DiMango et al., 1995). We detected a significant decrease in MIP-2 on day 1 in the garlic-extract-treated group $(P<0 \cdot 01)$. Since we see an inverse correlation between MIP-2 production and the presence of PMNs (although on different days), we hypothesize that the quorum-signal molecules (Bjarnsholt et al., 2005) short circuit the immune response. We have obtained similar results of elevated MIP-2 production by infecting mice with the wild-type $P$. aeruginosa, in contrast to the $\Delta l a s R$ rhlR QS mutant (unpublished results). When the QS system is blocked by garlic extract, an adequate immune response is mounted. The PMNs are more active, bacteria are efficiently eradicated, and in turn the bacterial stimulation of PMN recruiting cytokines is reduced. However, when the QS system is functioning, the production of MIP-2 is up-regulated but the PMNs are impaired; they do not accumulate at the site of infection and therefore do not counteract the bacteria properly. We base this hypothesis on the observation that for some of the garlic-treated mice the PMNs had migrated 
Table 1. Histopathological evaluation of types of leukocytes after garlic treatment

The types of leukocytes that were detected in $P$. aeruginosainfected mice lungs (after treatment with garlic extract or placebo) are presented. The results from three different days of sacrifice are shown, and whether the lung was dominated by PMNs, PMNs and MNs, or MNs, or whether no leukocytes were detected. On day 5, we detected a significant difference in the presence of PMNs, 13/14 garlic-treated mice had both PMNs and MNs present in the lung tissue. This is compared to day 5 of the placebotreated group of mice in which only 4 mice had both PMNs and MNs, and 6 had only MNs present. The rest of the lungs we scored as N.A.D., since no leukocytes were detected. The statistical difference between $13 / 14$ and $4 / 13$ was calculated by a $\chi^{2}$ test $(P<0 \cdot 0008)$.

\begin{tabular}{|llccc|}
\hline Treatment & $\begin{array}{c}\text { Type of } \\
\text { leukocytes }\end{array}$ & $\begin{array}{c}\text { Day } \\
\mathbf{1}\end{array}$ & $\begin{array}{c}\text { Day } \\
\mathbf{3}\end{array}$ & $\begin{array}{c}\text { Day } \\
\mathbf{5}\end{array}$ \\
\hline Placebo & & & & \\
& PMN & 5 & 1 & 0 \\
& PMN/MN & 2 & 5 & 4 \\
& MN & 0 & 0 & 6 \\
Garlic extract & N.A.D. & 0 & 1 & 3 \\
& PMN & 7 & 3 & 0 \\
& PMN/MN & 0 & 4 & 13 \\
& MN & 0 & 0 & 0 \\
& N.A.D. & 0 & 0 & 1 \\
Statistical evaluation & & & & \\
& & N.S. & N.S. & $P<0 \cdot 0008$ \\
\hline
\end{tabular}

N.S., Not significant; N.A.D., nothing abnormal detected.

into the bronchi as well as in the parenchyma, whereas in the placebo-treated mice the PMNs had only migrated into the parenchyma (data not shown).

We also detected a significantly lower production of G-CSF in the garlic-extract-treated group of mice on day $1(P<$ $0 \cdot 04)$ and day $3(P<0 \cdot 05)$. G-CSF, being a major mobilizer of PMNs, contributes to impaired lung function during the chronic $P$. aeruginosa lung infection in CF patients (Jensen, 2003). A reduction in G-CSF was shown to correlate with improved lung function. We link the reduced G-CSF production in the garlic-extract-treated group of mice to an adequate immune response brought about by the blocked QS system.

The higher levels of both MIP-2 and G-CSF seen in the untreated group of mice compared to the treated group of mice correlate to the state of chronic infection observed in CF patients. MPO was measured to determine the amount of PMNs present in the lungs (data not shown). No difference in MPO level was determined between the two groups, indicating that the same amount of PMNs is present in the lung homogenate despite the treatment. Since bacteria are cleared in the treated group, and we
Table 2. Histopathological evaluation of degree of inflammation after garlic treatment

The degree of inflammation in $P$. aeruginosa-infected mice lungs (after treatment with garlic extract or placebo) was accounted for by the number of leukocytes present in the lungs The inflammation was scored as 0 (no inflammation), 1 (mild inflammation), 2 (moderate to severe inflammation) and 3 (severe inflammation with necrosis or severe inflammation throughout the lung). The histopathological evaluation was performed blind. We detected a higher degree of inflammation in the garlic-treated group of mice as calculated by a Mann-Whitney test for nonparametric data $(P<0 \cdot 04)$.

\begin{tabular}{|lcccc|}
\hline Treatment & $\begin{array}{c}\text { Degree of } \\
\text { inflammation }\end{array}$ & $\begin{array}{c}\text { Day } \\
\mathbf{1}\end{array}$ & $\begin{array}{c}\text { Day } \\
\mathbf{3}\end{array}$ & $\begin{array}{c}\text { Day } \\
\mathbf{5}\end{array}$ \\
\hline Placebo & & & & \\
& 0 & 0 & 1 & 3 \\
& 1 & 4 & 0 & 7 \\
& 2 & 3 & 6 & 3 \\
Garlic extract & 3 & 0 & 0 & 0 \\
& & & & \\
& 0 & 0 & 0 & 1 \\
& 1 & 1 & 2 & 4 \\
& 2 & 6 & 5 & 9 \\
Statistical evaluation & 3 & 0 & 0 & 0 \\
& & & & \\
& & N.S. & N.S. & $P<0 \cdot 04$ \\
\hline
\end{tabular}

N.S., Not significant.

detect more PMNs inside the lungs, we hypothesize that QS signals might function to block the influx of PMNs into the lung. The extravasation of PMNs is possibly reduced in the untreated group and the PMNs are stuck to

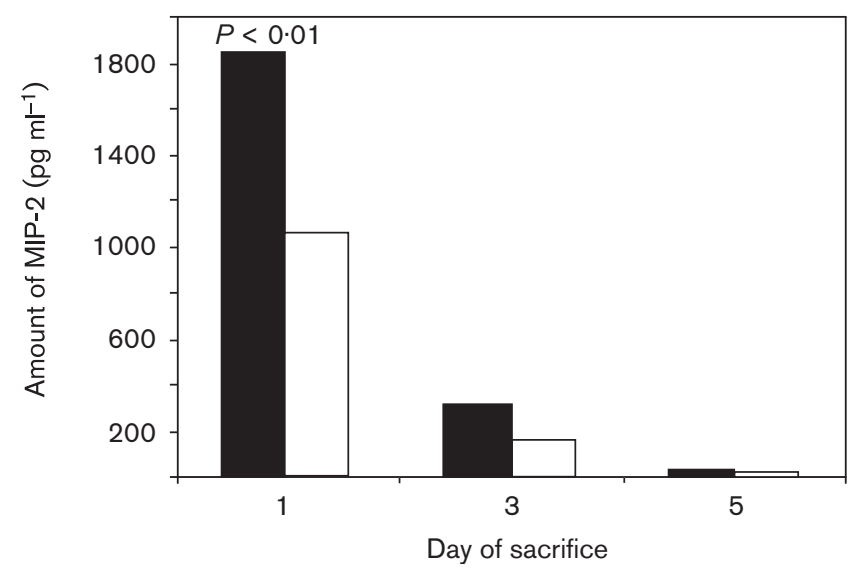

Fig. 4. Amount of MIP-2 present in the lung tissue. A higher level of MIP-2 was detected in the placebo (saline)-treated group of mice (black bars) on day 1 compared to the garlicextract-treated group of mice (white bars) $(P<0.01)$. 


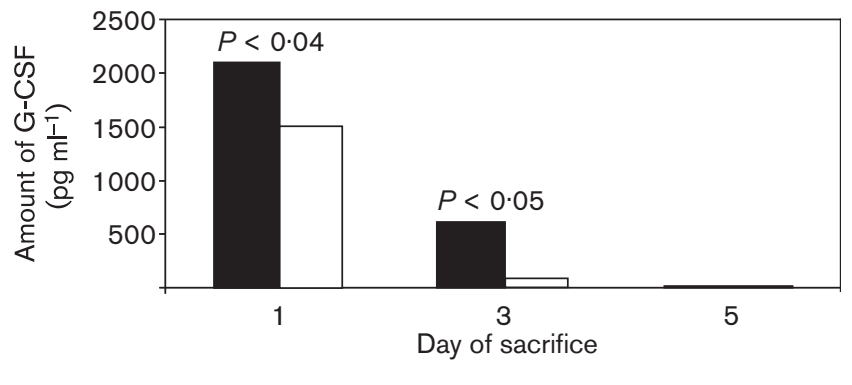

Fig. 5. Amount of G-CSF present in the lung tissue. A higher level of G-CSF was detected in the placebo (saline)-treated group of mice (black bars) on days 1 and 3 compared to the garlic-extract-treated group of mice (white bars), $(P<0.04)$ and $(P<0 \cdot 05)$, respectively.

endothelial cells where they cause collateral damage to the lung tissue.

The above cytokines were also measured in uninfected, garlic-treated mice, as well as uninfected, saline-treated mice. Neither saline nor garlic treatment alone leads to changes in cytokine production (data not shown). Similarly, neither inflammatory nor pathological changes were observed (data not shown).

\section{Conclusion}

The present report demonstrates that a QS-blocking garlic treatment is a possible approach to the attenuation of virulence and control of $P$. aeruginosa infections. Studies carried out at the Danish CF Center in Copenhagen (Ciofu et al., 1994) have shown the development of bacterial resistance to antibiotics to be a serious side-effect of the current anti-pseudomonal treatment, and it highlights the importance of the development of therapies that limit the formation of persistent biofilms in the lungs. A balance between colonization and clearance causes the slow development of chronic $P$. aeruginosa infection. When the balance is pushed in the direction of colonization, the biofilm mode of growth protects the colonizing bacteria from the host defence system and increases the tolerance to antibiotics. It is therefore interesting that QS-blocking drugs that efficiently eradicate pulmonary infections, in our animal model, also enable a more appropriate PMN response in the presence of $P$. aeruginosa biofilms. The administration of such drugs is expected not only to lead to the development of less persistent biofilms but also to inhibit the expression of bacterial virulence determinants that actively degrade components of the defence system (Kharazmi et al., 1986). Taken together with the synergistic effect of QS blockage and PMN activation, this might suffice to reverse the delicate balance in favour of the host clearance mechanism, and thereby reverse the severity of infection and improve the lung function. For younger CF individuals without chronic $P$. aeruginosa infections, an early prophylactic treatment based on these drugs might prevent the formation of persistent and damaging biofilms in the lung. However, in order to achieve the garlic-extract dose used for the treatment of the infectious mouse model, an $80 \mathrm{~kg}$ person would have to ingest 50 whole bulbs of garlic every day! Work is currently in progress to isolate and characterize the pure compounds responsible for blocking the $P$. aeruginosa QS systems.

\section{ACKNOWLEDGEMENTS}

This work was supported by grants from the Danish Technical Research Council and the Villum Kann-Rasmussen Foundation to M. G., and from the Cystic Fibrosis Foundation Therapeutics, Inc. and the German Mukoviszidose e.V. to M.G. and L.E. The technical assistance of Linda Stabell and Jette Pedersen was greatly appreciated.

\section{REFERENCES}

Ankri, S. \& Mirelman, D. (1999). Antimicrobial properties of allicin from garlic. Microbes Infect 1, 125-129.

Bassøe, C. F., Li, N., Ragheb, K., Lawler, G., Sturgis, J. \& Robinson, J. P. (2003). Investigations of phagosomes, mitochondria, and acidic granules in human neutrophils using fluorescent probes. Cytometry 51B, 21-29.

Bjarnsholt, T., Jensen, P. O., Burmølle, M. \& 9 other authors (2005). Pseudomonas aeruginosa tolerance to tobramycin, hydrogen peroxide and polymorphonuclear leukocytes is quorum-sensing dependent. Microbiology 151, 373-383.

Chmiel, J. F. \& Davis, P. B. (2003). State of the art: why do the lungs of patients with cystic fibrosis become infected and why can't they clear the infection? Respir Res 4, 8.

Christensen, B. B., Sternberg, C., Andersen, J. B., Palmer, R. J., Jr, Nielsen, A. T., Givskov, M. \& Molin, S. (1999). Molecular tools for study of biofilm physiology. Methods Enzymol 310, 20-42.

Ciofu, O., Giwercman, B., Pedersen, S. S. \& Høiby, N. (1994). Development of antibiotic resistance in Pseudomonas aeruginosa during two decades of antipseudomonal treatment at the Danish CF Center. APMIS 102, 674-680.

DiMango, E., Zar, H. J., Bryan, R. \& Prince, A. (1995). Diverse Pseudomonas aeruginosa gene products stimulate respiratory epithelial cells to produce interleukin-8. J Clin Invest 96, 2204-2210.

Fuqua, W. C., Winans, S. C. \& Greenberg, E. P. (1994). Quorum sensing in bacteria: the LuxR-LuxI family of cell density-responsive transcriptional regulators. J Bacteriol 176, 269-275.

Hentzer, M., Wu, H., Andersen, J. B. \& 15 other authors (2003). Attenuation of Pseudomonas aeruginosa virulence by quorum sensing inhibitors. EMBO J 22, 3803-3815.

Jensen, P. O. (2003). Characterization \& modulation of the innate immune response during Pseudomonas aeruginosa lung infection in patients with cystic fibrosis: an experimental \& clinical study. $\mathrm{PhD}$ thesis, University of Copenhagen.

Kharazmi, A., Eriksen, H. O., Doring, G., Goldstein, W. \& Høiby, N. (1986). Effect of Pseudomonas aeruginosa proteases on human leukocyte phagocytosis and bactericidal activity. Acta Pathol Microbiol Scand Sect C Immunol 94, 175-179.

Koch, C. \& Høiby, N. (2000). Diagnosis and treatment of cystic fibrosis. Respiration 67, 239-247.

Lyczak, J. B., Cannon, C. L. \& Pier, G. B. (2002). Lung infections associated with cystic fibrosis. Clin Microbiol Rev 15, 194-222.

Moser, C., Johansen, H. K., Song, Z., Hougen, H. P., Rygaard, J. \& Høiby, N. (1997). Chronic Pseudomonas aeruginosa lung infection is 
more severe in Th2 responding $\mathrm{BALB} / \mathrm{c}$ mice compared to Th1 responding $\mathrm{C} 3 \mathrm{H} / \mathrm{HeN}$ mice. APMIS 105, 838-842.

Moser, C., Hougen, H. P., Song, Z., Rygaard, J., Kharazmi, A. \& Høiby, N. (1999). Early immune response in susceptible and resistant mice strains with chronic Pseudomonas aeruginosa lung infection determines the type of T-helper cell response. APMIS 107, 1093-1100.

Moser, C., Kjaergaard, S., Pressler, T., Kharazmi, A., Koch, C. \& Høiby, N. (2000). The immune response to chronic Pseudomonas aeruginosa lung infection in cystic fibrosis patients is predominantly of the Th2 type. APMIS 108, 329-335.

Pedersen, S. S., Shand, G. H., Hansen, B. L. \& Hansen, G. N. (1990). Induction of experimental chronic Pseudomonas aeruginosa lung infection with $P$. aeruginosa entrapped in alginate microspheres. APMIS 98, 203-211.

Persson, T., Hansen, T. H., Rasmussen, T. B., Skindersø, M. E., Givskov, M. \& Nielsen, J. (2005). Rational design and synthesis of new quorum-sensing inhibitors derived from acylated homoserine lactones and natural products from garlic. Org Biomol Chem 3, 253-262.

Rasmussen, T. B., Bjarnsholt, T., Skindersoe, M. E., Hentzer, M., Kristoffersen, P., Köte, M., Nielsen, J., Eberl, L. \& Givskov, M. (2005). Screening for quorum-sensing inhibitors (QSI) by use of a novel genetic system, the QSI selector. J Bacteriol 187, 1799-1814.

van Delden, C. \& Iglewski, B. H. (1998). Cell-to-cell signaling and Pseudomonas aeruginosa infections. Emerg Infect Dis 4, 551-560.

Wu, H., Song, Z., Givskov, M., Doring, G., Worlitzsch, D., Mathee, K., Rygaard, J. \& Høiby, N. (2001). Pseudomonas aeruginosa mutations in lasI and rhlI quorum sensing systems result in milder chronic lung infection. Microbiology 147, 1105-1113.

Wu, H., Song, Z., Hentzer, M., Andersen, J. B., Molin, S., Givskov, M. \& Høiby, N. (2004). Synthetic furanones inhibit quorum-sensing and enhance bacterial clearance in Pseudomonas aeruginosa lung infection in mice. J Antimicrob Chemother 53, 1054-1061. 